\title{
Ortho and Parahydrogen in Interstellar Material
}

\author{
Robert R. Reeves and Paul Harteck \\ Department of Chemistry, Rensselaer Polytechnic Institute, Troy, New York
}

Z. Naturforsch. 34a, 163-166 (1979); received November 23, 1978 *

Since $\mathrm{H}_{2}$ is the most abundant molecule in the universe, the ratio of orthohydrogen molecules to parahydrogen in interstellar space is of interest. $\mathrm{H}_{2}$, formed by any exothermic reaction will be in the ratio of $3: 1$ according to their statistical weight. This corresponds to the high temperature equilibrium. At the low prevailing temperatures of interstellar space, the thermodynamic equilibrium should be shifted to the parahydrogen side. In the gas phase this shift can occur only over chemical reactions which are close to thermally neutral. In addition, it can be concluded that two ion scrambling reactions dominate the process and these both involve positive hydrogen ions. In special cases, surface catalysis on interstellar dust particles may add to the equilibrating process. The highly forbidden process of transition by radiation $J=1$ (ortho) $\rightarrow$ $J=0_{\text {(para) }}+h v$ can only play a minor role in this catalysis.

50 years ago experimental evidence could be given that normal hydrogen is composed of two forms which, under normal conditions, do not interchange because a very strong selection rule (perhaps the strongest, which exist in atom physics) forbids such an interchange. These two forms or modifications of hydrogen were termed ortho and parahydrogen [1]. The former was identified with the odd rotational states $(J=1,3,5 \ldots)$ the latter with the even states $(J=0,2,4 \ldots)$. Extensive studies of the ortho-parahydrogen system were made and an excellent early summary is found in the book of Farkas [2]. The energy difference between the $J=1$ of orthohydrogen and $J=0$ of parahydrogen is $0.34 \mathrm{kcal}$ [3]. At temperatures above $300 \mathrm{~K}$ the equilibrium ratio is practically 1 parahydrogen to 3 orthohydrogen. This ratio is due to the fact that in parahydrogen the spins of the two protons in the $\mathrm{H}_{2}$ molecule are antiparallel, whereas in orthohydrogen they are parallel. The thermal equilibrium, if established, is shifted to the parahydrogen with decreasing temperature (see Tables 1 and 2). Since the hydrogen molecule is by far the most abundant element in the universe, it is of interest to discuss the ratio of these $\mathrm{H}_{2}$ modifications in the interstellar environment.

In many regions of interstellar space hydrogen atoms are predominant over hydrogen molecules. In the dense clouds, however, molecular hydrogen is present in higher concentrations $[4,5]$, and in

\footnotetext{
* First version was received August 22, 1978.
}

Reprint requests to Prof. P. Harteck, Department of Chemistry, Rensselaer Polytechnic Institute, Troy/New York 12181.

$0340-4811 / 79 / 0200-0163 \$ 01.00 / 0$
Table $1 *$. Percent parahydrogen in equilibrium as a function of temperature.

\begin{tabular}{llll}
\hline$T$ & $\begin{array}{l}\text { Para- } \\
\text { hydrogen } \\
{[\%]}\end{array}$ & $\begin{array}{l}T \\
{[\mathrm{~K}]}\end{array}$ & $\begin{array}{l}\text { Para- } \\
\text { hydrogen } \\
{[\%]}\end{array}$ \\
\hline 10 & 100.0 & 100 & 38.5 \\
25 & 99.0 & 150 & 28.5 \\
50 & 76.9 & 200 & 26.0 \\
75 & 51.9 & 300 & 25.1 \\
\hline
\end{tabular}

* From Reference [3], page 14.

Table $2 *$. Distribution of hydrogen species at various temperatures.

\begin{tabular}{|c|c|c|c|c|c|}
\hline $\begin{array}{l}\text { Temper- } \\
\text { ature }[\mathbf{K}]\end{array}$ & Hydrogen & $J=0$ & $J=1$ & $J=2$ & $J=3$ \\
\hline \multirow[t]{4}{*}{50} & Equilibrium & 76.88 & 23.11 & 0.01 & - \\
\hline & Para & 99.98 & - & 0.02 & - \\
\hline & Ortho & - & 100.00 & - & - \\
\hline & Normal & 24.995 & 75.00 & 0.005 & - \\
\hline \multirow[t]{4}{*}{100} & Equilibrium & 37.36 & 61.46 & 1.15 & 0.02 \\
\hline & Para & 97.02 & - & 2.98 & - \\
\hline & Ortho & - & 99.95 & - & 0.05 \\
\hline & Normal & 24.25 & 74.97 & 0.74 & 0.03 \\
\hline \multirow[t]{4}{*}{200} & Equilibrium & 18.65 & 71.67 & 7.27 & 2.38 \\
\hline & Para & 71.82 & - & 28.05 & - \\
\hline & Ortho & - & 96.78 & - & 3.21 \\
\hline & Normal & 17.95 & 72.59 & 7.01 & 2.41 \\
\hline
\end{tabular}

* From Reference [3], page 15.

certain regions the molecular form may be present in amounts exceeding the hydrogen atom concentrations by orders of magnitude. The selection rule which forbids the transition from ortho to parahydrogen is very strict. Therefore a transition by radiation or interaction between hydrogen molecules at densities of the interstellar space or dark 
clouds cannot occur even in "aeons". The shift of ortho to parahydrogen can only occur at temperatures lower than $300 \mathrm{~K}$ and furthermore the reaction must be thermal or almost thermally neutral. Exothermic reactions are out of the question, because the exothermicity, $Q$, is normally orders of magnitude larger than the energy difference between the lowest levels of ortho and parahydrogen and $Q \gg 120 \mathrm{~cm}^{-1}$, or $0.34 \mathrm{kcal}$. Therefore in practically all chemical reactions even at low temperatures the ratio of ortho to parahydrogen produced will be $3: 1$. Two examples of exothermic processes which will result in the formation of hydrogen in the $3: 1$ ratio of ortho to para, corresponding to a high temperature equilibrium are:

(1) The formation process of $\mathrm{H}_{2}$ by three-body or surface recombination: $\mathrm{H}+\mathrm{H}+\mathrm{M}=\mathrm{H}_{2}+\mathrm{M}$;

(2) The catalytic recombination over electrons and negative ions according to the following mechanism:

a) $\mathrm{H}+\mathrm{e}^{-} \rightarrow \mathrm{H}^{-}+h v$

b) $\mathrm{H}^{-}+\mathrm{H} \rightarrow \mathrm{H}_{2}+\mathrm{e}^{-}-82$ kcal.

Hydrogen molecules on adsorbers with paramagnetic centers, however, may thermally equilibrate ortho-parahydrogen even at the lowest temperatures due to paramagnetic catalysis.

In the gas phase scrambling ion-reactions which equilibrate the ortho-para ratio to the ambient kinetic temperature seems to be by far the most effective ones, because the rates of these reactions are extremely fast and many appear to have no energy of activation.

The scrambling reaction involving a neutral hydrogen atom, namely

$$
\mathrm{H}_{2 \text { (ortho) }}+\mathrm{H} \rightarrow \mathrm{H}+\mathrm{H}_{2 \text { (thermal equilibrium) }} \text {, }
$$

has an energy of activation of about $7 \mathrm{kcal}$ [6] and the rate, therefore, becomes negligible at the low temperature of interstellar space. The ion reactions

$$
\mathrm{H}_{2(\text { ortho) }}+\mathrm{H}^{+} \rightarrow \mathrm{H}^{+}+\mathrm{H}_{2 \text { (thermal equilibrium) }}
$$

and

$$
\mathrm{H}_{2 \text { (ortho) }}+\mathrm{H}_{3}{ }^{+} \rightarrow \mathrm{H}_{3}{ }^{+}+\mathrm{H}_{2 \text { (thermal equilibrium) }}
$$

should have very high rate constants and little or no energy of activation. All are of a catalytic nature and they are exothermic only due to the loss of one rotational quantum by the ortho-para conversion.
For the reaction

$$
\mathrm{H}_{2}{ }^{+}+\mathrm{H}_{2}=\mathrm{H}_{3}{ }^{+}+\mathrm{H},
$$

the rate constant has been determined $[7,8]$ to be $k_{4}=2.1 \times 10^{-9} \mathrm{~cm}^{3} / \mathrm{sec}$. The rate constants $k_{2}$ and $k_{3}$ have not been measured, but should be similar to that for reaction (4). There are furthermore many analogous ion reactions cited by Herbst and Klemperer [9], including $\mathrm{H}_{3}{ }^{+}$interacting with $\mathrm{H}_{2} \mathrm{O}, \mathrm{NH}_{3}, \mathrm{OH}$, with rate constants of about $2 \times 10^{-9} \mathrm{~cm}^{3} / \mathrm{sec}$. Huntress [10] also cites a large number of ion reactions and the corresponding rates, and has measured $\mathrm{H}_{3}{ }^{+}+\mathrm{HD} \rightarrow \mathrm{H}_{2} \mathrm{D}^{+}+\mathrm{H}_{2}$, which is analogous to reaction (3), and found $k=0.3 \times 10^{-9}$. This reaction is expected to be slightly endothermic, and reaction (3) should be at least twice as fast, since either $\mathrm{H}$-atom of the $\mathrm{H}_{2}$ may interact with the $\mathrm{H}_{3}{ }^{+}$. The rate then is approximately $10^{-9} \mathrm{~cm}^{3} / \mathrm{sec}$.

Many estimates have been made of the concentrations of $\mathrm{H}^{+}$and $\mathrm{H}_{3}{ }^{+}$ions as well as $\mathrm{H}_{2}{ }^{+}, \mathrm{H}$, and $\mathrm{H}_{2}$ in the regions of interstellar space. Values for $\left|\mathrm{H}^{+}\right|$of $10^{-3}$ and for $\left|\mathrm{H}_{3}+\right|$ of $10^{-6} \mathrm{~cm}^{-3}$ are typical. Although the $\mathrm{H}^{+}$and $\mathrm{H}_{3}{ }^{+}$are primarily formed in most instances as "hot" ions in strongly exothermic reactions, the rate of thermalization for these "hot" ions can be estimated, and one may conclude that after about 10 collisions the hot ions with an initial energy of a few $\mathrm{eV}$ will be thermalized and after this a long chain $\left(10^{3}\right.$ to $10^{6}$, depending on conditions) of $\mathrm{H}_{2 \text { (equilibrium) }}$ scrambling steps will occur. Since the concentration of $\mathrm{H}^{+}$is orders of magnitude larger than $\mathrm{H}_{3}+$ in interstellar space, most of the scrambling should occur over reaction (2). Assuming $k_{2}$ and $k_{3}$ are both on the order of $10^{-9} \mathrm{~cm}^{3} / \mathrm{sec}$, the probability $\Sigma$ for a hydrogen molecule undergoing conversion should be in the order of $10^{-12} / \mathrm{sec}$ :

scrambling $=\left|\mathrm{H}^{+}\right| k_{2}+\left|\mathrm{H}_{3}{ }^{+}\right| k_{3}$ probability $=\sim\left|10^{-3}\right| 10^{-9} / \mathrm{sec}+\left|10^{-6}\right| 10^{-9} / \mathrm{sec}$.

A probability of $10^{-12} / \mathrm{sec}$ means that scrambling should occur once in $10^{12}$ seconds or about 30,000 years. The probability of a $\mathrm{H}_{2}$-molecule being ionized, whereby its symmetry becomes destroyed, however is only between $10^{-18}$ and $10^{-15} \mathrm{sec}^{-1}$.

The scrambling probabilities may be readily estimated for various molecular hydrogen densities, since Herbst and Klemperer [9] have calculated and tabulated $\mathrm{H}^{+}$and $\mathrm{H}_{3}{ }^{+}$concentrations for 
cosmic ray induced ionization of $\mathrm{H}_{2}$, as a function of $\mathrm{H}_{2}$ density. The ionization probability for $\mathrm{H}_{2}$ was assumed to be $(\zeta)=10^{-17} \mathrm{sec}^{-1}$. Practically all possible reactions and rates had been considered and the simultaneous coupled nonlinear equations were solved numerically. Here only the stationary concentrations of $\mathrm{H}^{+}$and $\mathrm{H}_{3}{ }^{+}$are quoted for $\mathrm{H}_{2}$ concentrations of $10^{4}, 10^{5}$ and $10^{6} \mathrm{~cm}^{-3}$. The scrambling probability is given in Table 3 as calculated by the relation:

$$
\text { Scrambling/sec }=k_{2}\left|\mathrm{H}^{+}\right|+k_{3}\left|\mathrm{H}_{3}{ }^{+}\right|
$$

with $k_{2}=k_{3}=\sim 10^{-9} \mathrm{~cm}^{3} / \mathrm{sec}$.

Table 3. Ion densities and associated ortho-parahydrogen scrambling probabilities for various hydrogen densities.

\begin{tabular}{llll}
\hline & $\mathrm{cm}^{-3}$ & $\mathrm{~cm}^{-3}$ & $\mathrm{~cm}^{-3}$ \\
\hline$\left|\mathrm{H}_{2}\right|$ & $1 \cdot 10^{4}$ & $1 \cdot 10^{5}$ & $1 \cdot 10^{6}$ \\
$\mathrm{H}^{+} \mid$ & $2 \cdot 10^{-4}$ & $7 \cdot 10^{-4}$ & $5 \cdot 10^{-3}$ \\
$\mathrm{H}_{3}{ }^{+} \mid$ & $7 \cdot 10^{-6}$ & $7 \cdot 10^{-6}$ & $7 \cdot 10^{-6}$ \\
scrambling/sec & $2 \cdot 10^{-13}$ & $7 \cdot 10^{-13}$ & $5 \cdot 10^{-12}$ \\
scrambling & & & \\
time in sec & $5 \cdot 10^{12}$ & $1.4 \cdot 10^{12}$ & $2 \cdot 10^{11}$ \\
\hline
\end{tabular}

According to this table a scrambling will occur in 6,300 years at a hydrogen concentration of $10^{6} \mathrm{~cm}^{-3}$, and in 160,000 years at a concentration of $10^{4} \mathrm{~cm}^{-3}$. These scrambling data are in line with the rough estimate made above where $\mathrm{H}^{+}$densities of $10^{-3} \mathrm{~cm}^{-3}$ were assumed. It should be noted that the scrambling by the $\mathrm{H}^{+}$is dominant.

The competitive scrambling process by (2) and (3), however, may change substantially relative to each other under special conditions, if the $\mathrm{H}_{3}{ }^{+}$concentration exceeds the $\mathrm{H}^{+}$concentration. A specific example with a relatively high oxygen concentration was calculated by Herbst and Klemperer [9], where $\mathrm{H}^{+}=2 \times 10^{-6}$ and $\mathrm{H}_{3}{ }^{+}=7 \times 10^{-6} \mathrm{~cm}^{-3}$, and there the scrambling probability would be $\sim 10^{-14}$ sec. This is equivalent to a relaxation time of $\sim 3 \times 10^{6}$ years. With such long relaxation times, we may reach the limit of time available, due to the limited lifetime of the dense clouds (in the order of a million years), for a quantitative scrambling. With this in mind, it follows that there may exist regions in interstellar space, where the composition of the clouds is such that the relaxation time is larger than the time interval available for the thermal equilibration of the ortho-parahydrogen system.

Since many dark clouds are at kinetic temperatures of $\sim 20 \mathrm{~K}$ or somewhat higher, it may now be concluded that virtually all the molecular hydrogen, equilibrated over the scrambling reaction (2) and (3), is present as parahydrogen in the $J=0$ quantum state. (The equilibration data are given in Tables 2 and 3 for a few temperatures [2].)

The ortho-hydrogen molecules still present in space may be largely in the $J=1$ state. The radiation lifetime for the transition

$$
\mathrm{H}_{2}(J=1) \rightarrow \mathrm{H}_{2}(J=0)+h v
$$

has been first estimated by Wigner [12] as some hundreds of years. Theoreticists now indicate this radiative lifetime is orders of magnitude larger. Therefore the scrambling reaction by hydrogen ions should dominate over any radiative process.

As to our present knowledge there are no other possible scrambling reactions which could compete with reactions (2) or (3). The number of collisions with paramagnetic molecules or particles like $\mathrm{O}_{2}$, radicals, electrons and hydrogen atoms would be insufficient, by many orders of magnitude, to cause significant interconversion.

The number of electrons and negatively charged ions, especially $\mathrm{H}^{-}$, must be the same essentially as the $\mathrm{H}^{+}$and $\mathrm{H}_{3}{ }^{+}$for electrical neutrality. The scrambling could occur over two main reactions, namely

$$
\mathrm{H}^{-}+\mathrm{H}_{2 \text { (ortho) }} \rightarrow \mathrm{H}_{2 \text { (para) }}+\mathrm{H}^{-},
$$

and

$$
\mathrm{e}^{-}+\mathrm{H}_{2 \text { (ortho) }} \rightarrow \mathrm{H}_{2 \text { (para) }}+\mathrm{e}^{-} .
$$

The $\mathrm{H}^{-}$steady-state concentration is negligible, however, since the attachment rate of the electron on to the $\mathrm{H}$-atom is very slow and the detachment due to interaction with the $\mathrm{H}$-atoms is very fast:

$$
\mathrm{H}^{-}+\mathrm{H} \rightarrow \mathrm{H}_{2}+\mathrm{e}^{-} \text {. }
$$

Reaction (7) must be very slow, since the electron can not accept the appropriate momentum. This has long been known experimentally since even a weak electric discharge can be used to observe the ortho-parahydrogen distribution in the emission from the excited molecules [1].

It should be mentioned that the scrambling reaction (2) should, by far, be the most abundant "chemical reaction" which occurs in the universe. 
A systematic measurement of the ratio of ortho to para $\mathrm{H}_{2}$ in the universe would add considerably to our overall knowledge of interstellar space.

It is of interest to determine if deuterium could interfer in the scrambling process. In this case, the deuterium ion, $\mathrm{D}^{+}$, has an ionization potential which is about $29.8 \mathrm{~cm}^{-1}$ or $85.9 \mathrm{cal}$ higher than the ionization potential of $\mathrm{H}^{+}$. Therefore the collision between an $\mathrm{H}$ and $\mathrm{D}$ from which one is ionized will interact with a Boltzmann factor of

$$
\exp \{(+85.9) / R T / \mathrm{cal}\}
$$

in favor of the formation of $\mathrm{H}^{+}$. Therefore, in interstellar space, depending upon the kinetic temperature of the particles, this Boltzmann factor can become substantial and $\mathrm{H}+\mathrm{D}^{+} \rightarrow \mathrm{H}^{+}+\mathrm{D}$ occurs almost exclusively in the forward direction. The zero point-energy of the $\mathrm{H}_{2}$ molecule is larger than that of the HD molecule, which is in turn larger than that of the $\mathrm{D}_{2}$. Therefore a collision between $\mathrm{H}^{+}$and $\mathrm{HD}$ will not result in a "chemical reaction". However $\mathrm{D}^{+}$will interact with $\mathrm{H}_{2}$ or $\mathrm{HD}$, producing $\mathrm{H}^{+}$:

$$
\mathrm{D}^{+}+\mathrm{H}_{2}=\mathrm{HD}+\mathrm{H}^{+}-0.9 \mathrm{kcal}
$$

[1] K. F. Bonhoeffer and P. Harteck, Z. Phys. Chem. B 4, 113 (1929).

[2] A. Farkas, Orthohydrogen, Parahydrogen and Heavy Hydrogen, Cambridge University Press, Cambridge 1935.

[3] G. Herzberg, Spectra of Diatomic Molecules, Van Nostrand, Princeton, N. J. 1950, 2nd Ed., p. 532.

[4] D. J. Hollenback, M. W. Werner, and E. E. Salpeter, Astrophysical J. 163, 165 (1971).

[5] D. M. Rank, C. H. Townes, and W. J. Welch, Science 174, 1083 (1971). and

$$
\mathrm{D}^{+}+\mathrm{HD}=\mathrm{D}_{2}+\mathrm{H}^{+}-1.1 \mathrm{kcal} .
$$

(With the exothermicity of the reactions calculated for no rotational contribution from the molecules.)

Therefore, deuterium cannot interfere with the long scrambling chain to form parahydrogen.

Even though presently no measurements of the ratio of ortho to parahydrogen in interstellar space do exist, there should be, in the not too far future, the possibility to perform such measurements. Recently Smith [11] had discussed the orthoparahydrogen problem for the big outer planets: Jupiter, Saturn, Uranus and Neptune. In such dense hydrogen atmospheres "unsaturated $\mathrm{H}_{2}$ quadruple transitions" can be observed.

\section{Acknowledgement}

This research was supported by the Planetary Atmospheres program of the National Aeronautics and Space Administration under Grant No. NGL 33-018-007.

[6] See p. 69 of the book of Farkas.

[7] M. T. Bowers and D. D. Elleman, J. Chem. Phys. 50, 4787 (1969).

[8] S. O. Thompson and O. A. Schaefer, J. Amer. Chem. Soc. 80, 553 (1958).

[9] E. Herbst and W. Klemperer, Astrophysical J. 185, 505 (1973).

[10] W. Huntress, Astrophysical J., Suppl. Series 33, 495 (1977).

[11] W. H. Smith, Icarus 33, 210 (1978).

[12] E. Wigner, comments in [1], p. 126. 